\title{
Differential equations for loop integrals in Baikov representation
}

\author{
Jorrit Bosma, ${ }^{1, *}$ Kasper J. Larsen, ${ }^{2, \dagger}$ and Yang Zhang ${ }^{1,3, \$}$ \\ ${ }^{1}$ ETH Zürich, Wolfang-Pauli-Strasse 27, 8093 Zürich, Switzerland \\ ${ }^{2}$ School of Physics and Astronomy, University of Southampton, Highfield, \\ Southampton SO17 1BJ, United Kingdom \\ ${ }^{3}$ PRISMA Cluster of Excellence, Johannes Gutenberg University, 55128 Mainz, Germany
}

(Received 18 December 2017; published 18 May 2018)

\begin{abstract}
We present a proof that differential equations for Feynman loop integrals can always be derived in Baikov representation without involving dimension-shift identities. We moreover show that in a large class of two- and three-loop diagrams it is possible to avoid squared propagators in the intermediate steps of setting up the differential equations.
\end{abstract}

DOI: 10.1103/PhysRevD.97.105014

\section{INTRODUCTION}

The physics program of the Large Hadron Collider (LHC) demands precision calculations of production cross sections in order to have accurate predictions of the standard model signals and background. To match the experimental precision and the parton distribution function uncertainties, this requires for most processes computations at next-to-nextto leading order (NNLO) in fixed-order perturbation theory.

Out of the contributions to NNLO cross sections, the double-virtual one, i.e. the two-loop scattering amplitude, is the main theoretical bottleneck. Two-loop amplitudes for most $2 \rightarrow 2$ processes of phenomenological interest have now been computed [1-25]. Results for $2 \rightarrow n$ processes with $n>2$ are largely restricted to all-plus helicity amplitudes [26-33], though impressive results on all helicity amplitudes for the planar five-gluon amplitude have recently appeared [34]. Expressions for the planar fivegluon basis integrals were computed in Refs. [28,35].

Calculation of multiloop amplitudes proceeds in two stages. In the first step, the expression for the amplitude obtained from the Feynman rules after tensor reduction is recast as a linear combination of a basis of integrals, which leads to a significant reduction in the number of contributing integrals. (The existence of a finite basis was proved in Ref. [36].) This step is performed by use of integration-byparts (IBP) reductions. These arise from the vanishing integration of total derivatives in dimensional regularization,

\footnotetext{
jbosma@itp.phys.ethz.ch

Kasper.Larsen@ soton.ac.uk

zhang@uni-mainz.de
}

Published by the American Physical Society under the terms of the Creative Commons Attribution 4.0 International license. Further distribution of this work must maintain attribution to the author(s) and the published article's title, journal citation, and DOI. Funded by SCOAP.

$$
\int \prod_{i=1}^{L} \frac{\mathrm{d}^{D} \ell_{i}}{\mathrm{i} \pi^{D / 2}} \sum_{j=1}^{L} \frac{\partial}{\partial \ell_{j}^{\mu}} \frac{v_{j}^{\mu} P}{D_{1}^{\alpha_{1}} \cdots D_{m}^{\alpha_{m}}}=0
$$

where $P$ and the vectors $v_{j}^{\mu}$ are polynomial in the internal and external momenta, the $D_{k}$ denote inverse propagators, and the $\alpha_{i}$ are integers. In practice, the IBP identities generate a large set of linear relations between loop integrals which allows most of them to be reexpressed in terms of a set of basis integrals. The step of solving the linear systems that arise from Eq. (1.1) may be carried out by Gaussian elimination in the form of the Laporta algorithm [37,38], leading in general to relations that involve integrals with squared propagators. There are several publicly available implementations of automated IBP reduction: AIR [39], FIRE [40,41], Reduze [42,43], LiteRed [44], Kira [45], along with private implementations. A formalism for deriving IBP reductions without squared propagators was developed in Refs. [46,47]. A systematic way of deriving IBP reductions on generalizedunitarity cuts was given in Ref. [48]. A recent approach [49] uses sampling of finite-field elements to construct the reduction coefficients.

In the second step of computing a multiloop amplitude, one sets up differential equations satisfied by the basis integrals [50-58]. Letting $x_{m}$ denote an external kinematical invariant, $\epsilon=\frac{4-D}{2}$ the dimensional regulator, and $\mathcal{I}(\boldsymbol{x}, \epsilon)=\left(\mathcal{I}_{1}(\boldsymbol{x}, \epsilon), \ldots, \mathcal{I}_{N}(\boldsymbol{x}, \epsilon)\right)$ the basis of integrals, the result of differentiating any basis integral with respect to $x_{m}$ can be expressed as a linear combination of the basis integrals, once again by use of the IBP reductions. In this way, one finds a first-order linear system of partial differential equations,

$$
\frac{\partial}{\partial x_{m}} \mathcal{I}(\boldsymbol{x}, \epsilon)=A_{m}(\boldsymbol{x}, \epsilon) \mathcal{I}(\boldsymbol{x}, \epsilon)
$$


The boundary conditions can be fixed by using known results for a (typically small) subset of the simpler basis integrals and furthermore imposing regularity conditions, such as requiring vanishing discontinuities in certain kinematic channels. Having determined appropriate boundary conditions, the differential equations (1.2) can be solved to produce expressions for the basis integrals. In practice, this method has proven to be a powerful tool for computing multiloop integrals, enabling for example the calculation of the basis integrals for the $2 \rightarrow 2$ two-loop amplitudes mentioned above.

An important insight for the latter calculations was the observation [55] that in many cases (namely those where the basis integrals evaluate into multiple polylogarithms), the basis can be chosen such that the $\epsilon$-dependence factors out of the coefficient matrix, $A_{m}(\boldsymbol{x}, \epsilon)=\epsilon \hat{A}_{m}(\boldsymbol{x})$. In such a basis, often referred to as a canonical basis, integrating the differential equations in a Laurent expansion around $\epsilon=0$ becomes trivial.

In this paper we study differential equations of the type in Eq. (1.2) in the context of the IBP reduction formalisms of Refs. [46-48,59]. The main idea of the latter is to find appropriate choices of $v_{j}^{\mu}\left(\ell_{i}\right)$ in Eq. (1.1) for which the resulting IBP identities do not involve squared propagators. These IBP identities have the advantage of involving a more limited set of integrals than generic IBP identities, and as a result produce smaller linear systems to be solved.

The aim of this paper is two-fold. First, we prove that the differential equations (1.2) can be derived in Baikov representation, used in several recent papers [48,60-62], without involving dimension-shift identities. This is advantageous because the latter are computationally intensive to generate. Second, we investigate to what extent the IBP reduction formalism discussed in the previous paragraph is compatible with the differential equations (1.2). We will show that, for a large class of diagrams, it is possible to avoid having the derivatives on the left-hand side of Eq. (1.2) produce integrals with squared propagators. The obtained differential equations can subsequently be put in a canonical form $A_{m}(\boldsymbol{x}, \epsilon)=\epsilon \hat{A}_{m}(\boldsymbol{x})$ by applying the Lee-Moser algorithm [63-65] in single-scale cases, or multiscale generalizations [66]. The algorithms find a change-of-basis matrix to a canonical basis. They have been implemented in the publicly available codes EPSILON [67] and FUCHSIA [68], and CANONICA [69], respectively.

This paper is organized as follows. In Sec. II we set up notation and give the Baikov representation of a generic loop integral. In Sec. III we derive differential equations in Baikov representation and show that dimension shifts can be avoided provided that a certain ideal membership condition holds. In Sec. IV we give a general proof of the latter ideal membership. In Sec. V we show that if a stronger ideal membership holds, then integrals with squared propagators can be avoided in intermediate stages. In Sec. VI we give several examples where the stronger ideal membership is found to hold, along with the explicit differential equations. We also give a counterexample. In Sec. VII we give our conclusions. In the Appendix we derive differential equations in Baikov representation via dimension-shift identities.

\section{BAIKOV REPRESENTATION}

In this section we set up notation and introduce the Baikov representation of a generic Feynman loop integral.

We consider an $L$-loop integral with $k$ propagators and $m-k$ irreducible scalar products (i.e., polynomials in the loop momenta and external momenta which cannot be expressed as a linear combination of the inverse propagators). We work in dimensional regularization and normalize the integral as follows,

$$
I\left(N ; \alpha_{1}, \ldots, \alpha_{m} ; D\right) \equiv \int \prod_{j=1}^{L} \frac{\mathrm{d}^{D} \ell_{j}}{\pi^{D / 2}} \frac{N}{D_{1}^{\alpha_{1}} \cdots D_{m}^{\alpha_{m}}} .
$$

Here $N$ denotes a polynomial in the external momenta $p_{1}, \ldots, p_{E}, p_{E+1}$ and the loop momenta $\ell_{1}, \ldots, \ell_{L}$. The propagators are labeled so that

$$
\begin{aligned}
& \alpha_{i} \geq 1 \quad \text { for } \quad i=1, \ldots, k \\
& \alpha_{i} \leq 0 \quad \text { for } \quad i=k+1, \ldots, m .
\end{aligned}
$$

We remark that the notation (2.1) does not give a unique representation, since $D_{k+1}^{-\alpha_{k+1}} \cdots D_{m}^{-\alpha_{m}}$ can also be absorbed into $N$ to form a polynomial numerator,

$I(N ; \alpha ; D)=I\left(N \prod_{j=k+1}^{m} D_{j}^{-\alpha_{j}} ;\left(\alpha_{1}, \ldots, \alpha_{k}, \mathbf{0}\right) ; D\right)$.

Nevertheless, we find it more convenient to use this notation than to fix the projective invariance.

Our aim is now to present the Baikov representation [70] of the integral (2.1). To this end, we start by taking the set of all independent external and loop momenta,

$$
\left\{v_{1}, \ldots, v_{E+L}\right\}=\left\{p_{1}, \ldots, p_{E}, \ell_{1}, \ldots, \ell_{L}\right\},
$$

and form their Gram matrix $S$,

$$
S=\left(\begin{array}{ccc|ccc}
x_{1,1} & \cdots & x_{1, E} & x_{1, E+1} & \cdots & x_{1, E+L} \\
\vdots & \ddots & \vdots & \vdots & \ddots & \vdots \\
x_{E, 1} & \cdots & x_{E, E} & x_{E, E+1} & \cdots & x_{E, E+L} \\
\hline x_{E+1,1} & \cdots & x_{E+1, E} & x_{E+1, E+1} & \cdots & x_{E+1, E+L} \\
\vdots & \ddots & \vdots & \vdots & \ddots & \vdots \\
x_{E+L, 1} & \cdots & x_{E+L, E} & x_{E+L, E+1} & \cdots & x_{E+L, E+L}
\end{array}\right),
$$


where,

$$
x_{i j}=v_{i} \cdot v_{j} .
$$

It will be convenient for the derivations to follow to relabel the entries of the upper-left $E \times E$ block of $S$,

$$
\lambda_{i j}=x_{i j} \quad \text { for } \quad 1 \leq i, j \leq E,
$$

to emphasize that they are formed out of the external momenta only. Furthermore, it is useful to define the Gram matrix $G$ of the independent external momenta,

$$
G=\left(\begin{array}{ccc}
\lambda_{1,1} & \cdots & \lambda_{1, E} \\
\vdots & \ddots & \vdots \\
\lambda_{E, 1} & \cdots & \lambda_{E, E}
\end{array}\right)
$$

The entries of the remaining blocks of $S$ involve the loop momenta. Because $S$ is a symmetric matrix, the entries of the upper-right $E \times L$ block along with the upper-triangular entries of the lower-right $L \times L$ block,

$x_{i j}$ where $\left\{\begin{array}{l}1 \leq i \leq E \text { and } E+1 \leq j \leq E+L, \\ E+1 \leq i \leq j \leq E+L,\end{array}\right.$

are independent. As each set contributes respectively $L E$ and $\frac{L(L+1)}{2}$ elements, we find that the number of independent entries of $S$ that depend on the loop momenta is $L E+\frac{L(L+1)}{2}$. Since any inverse propagator $D_{\alpha}$ can be written as a unique linear combination of the $x_{i j}$, we can thus conclude that the combined number of propagators and irreducible scalar products in Eqs. (2.1) and (2.2) is given by,

$$
m=L E+\frac{L(L+1)}{2} .
$$

In particular, we have for $\alpha=1, \ldots, m$ that,

$$
D_{\alpha}=\sum_{\beta=1}^{m} A_{\alpha \beta} x_{\beta}+\sum_{1 \leq i \leq j \leq E}\left(B_{\alpha}\right)_{i j} \lambda_{i j} \quad \text { with } \quad A_{\alpha \beta} \in \mathbb{Z},
$$

where $\beta=1, \ldots, m$ labels the lexicographically-ordered elements $(i, j)$ in Eq. (2.9), and where the entries of $B_{\alpha}$ are integers.

The Baikov representation uses the inverse propagators and irreducible scalar products as variables,

$$
z_{\alpha} \equiv D_{\alpha} \quad \text { where } \quad 1 \leq \alpha \leq m
$$

The Jacobian associated with the change of variables from $\left(\ell_{1}^{\mu}, \ldots, \ell_{L}^{\mu}\right)$ in Eq. (2.1) to $\left(z_{1}, \ldots, z_{m}\right)$ is an appropriate power of the determinant of $S$,

$$
F \equiv \operatorname{det} S
$$

The Baikov representation of the integral in Eq. (2.1) takes the form [71],

$I(N ; \alpha ; D)=C_{E}^{L}(D) U^{\frac{E-D+1}{2}} \int \frac{\mathrm{d} z_{1} \cdots \mathrm{d} z_{m}}{z_{1}^{\alpha_{1}} \cdots z_{m}^{\alpha_{m}}} F^{\frac{D-L-E-1}{2}} N$,

where the first prefactor is given by,

$$
C_{E}^{L}(D) \equiv \frac{\pi^{-L(L-1) / 4-L E / 2}}{\prod_{j=1}^{L} \Gamma\left(\frac{D-L-E+j}{2}\right)} \operatorname{det} A,
$$

with $A$ defined in Eq. (2.11), and where $U$ denotes the determinant of the Gram matrix (2.8) of the independent external momenta,

$$
U \equiv \operatorname{det} G
$$

We remark that $U$ is equal to the square of the volume of the parallelotope formed by the independent external momenta $\left\{p_{1}, \ldots, p_{E}\right\}$ and therefore is nonvanishing for noncollinear external momenta.

\section{DIFFERENTIAL EQUATIONS IN BAIKOV REPRESENTATION}

In this section we show how differential equations of the form in Eq. (1.2) can be derived in Baikov representation. We note that related work has appeared in Refs. [60-62].

Letting $\left(I_{1}, \ldots, I_{M}\right)$ denote a basis of integrals and acting on the Baikov represention (2.14) by a derivative with respect to an arbitrary external invariant $\chi$, we find [72],

$$
\begin{aligned}
\frac{\partial}{\partial \chi} I_{j}\left(N_{j} ; \alpha ; D\right)= & \frac{E-D+1}{2 U} \frac{\partial U}{\partial \chi} I_{j}\left(N_{j} ; \alpha ; D\right) \\
& +\frac{D-L-E-1}{2} I_{j}\left(\frac{1}{F} \frac{\partial F}{\partial \chi} N_{j} ; \alpha ; D\right) .
\end{aligned}
$$

We observe that the $\frac{1}{F}$ factor in the second line effectively modifies the integration measure in Eq. (2.14), shifting the space-time dimension from $D$ to $D-2$.

This motivates us to ask whether there exist polynomials $\left(a_{1}, \ldots, a_{m}, b\right)$ such that the following relation holds,

$$
\frac{\partial F}{\partial \chi}=\sum_{i=1}^{m} a_{i} \frac{\partial F}{\partial z_{i}}+b F,
$$


since, as we will show shortly, this implies that dimension shifts can be avoided in Eq. (3.1). In the Appendix we work out the form of the differential equations (3.1) when $\frac{\partial F}{\partial \chi}$ is left unchanged by applying dimension-shift identities-i.e., relations between integrals in $D$ and $D-2$ dimensions.

The relation (3.2) can equivalently be stated as the ideal membership property,

$$
\frac{\partial F}{\partial \chi} \in\left\langle\frac{\partial F}{\partial z_{1}}, \ldots, \frac{\partial F}{\partial z_{m}}, F\right\rangle,
$$

where the ideal is understood to be embedded in the ring $\mathbb{C}\left[z_{1}, \ldots, z_{m}, \lambda_{i j}\right]$ of polynomials in the Baikov variables $\left(z_{1}, \ldots, z_{m}\right)$ and external invariants $\left(\lambda_{i, j}\right)_{i, j=1, \ldots, E}$ over $\mathbb{C}$.

In the following we will refer to Eq. (3.3) as the fundamental ideal membership. In Sec. IV we give a proof of Eq. (3.3). The proof is constructive and gives an explicit construction of the cofactors $\left(a_{1}, \ldots, a_{m}, b\right)$ in Eq. (3.2).

Let us now turn to the consequence of the ideal membership for the differential equations. Inserting Eq. (3.2) into Eq. (3.1) and using elementary integration by parts in $z_{i}$,

$\frac{a_{i} N_{j}}{z_{1}^{\alpha_{1}} \cdots z_{m}^{\alpha_{m}}} \frac{\partial}{\partial z_{i}} F^{\frac{D-L-E-1}{2}}=-\frac{F^{\frac{D-L-E-1}{2}}}{z_{1}^{\alpha_{1}} \cdots z_{m}^{\alpha_{m}}} z_{i}^{\alpha_{i}} \frac{\partial}{\partial z_{i}} \frac{a_{i} N_{j}}{z_{i}^{\alpha_{i}}}$,

we find,

$$
\begin{aligned}
\frac{\partial}{\partial \chi} I_{j}\left(N_{j} ; \alpha ; D\right)= & \frac{E-D+1}{2 U} \frac{\partial U}{\partial \chi} I_{j}\left(N_{j} ; \alpha ; D\right) \\
& +I_{j}\left(Q_{j} ; \alpha ; D\right)
\end{aligned}
$$

where the insertion in the second term is given by,

$Q_{j}=-\sum_{i=1}^{m} z_{i}^{\alpha_{i}} \frac{\partial}{\partial z_{i}}\left(\frac{a_{i} N_{j}}{z_{i}^{\alpha_{i}}}\right)+\frac{D-L-E-1}{2} b N_{j}$.

We observe that all integrals in the differential equations (3.5) are $D$-dimensional integrals, so that no dimension-shift identities are required.

By use of integration-by-parts reductions we can express the integrals in the second line of Eq. (3.5) as linear combinations of the basis integrals $I_{j}\left(N_{j} ; \alpha ; D\right)$,

$$
I_{j}\left(Q_{j} ; \alpha ; D\right)=\sum_{k=1}^{M} R_{j k}(\lambda, D) I_{k}\left(N_{k} ; \alpha ; D\right) .
$$

Thus, we arrive at the system of differential equations,

$$
\frac{\partial}{\partial \chi} I_{j}\left(N_{j} ; \alpha ; D\right)=\sum_{k=1}^{M} A_{j k}(\lambda, D) I_{k}\left(N_{k} ; \alpha ; D\right),
$$

where the coefficient matrix is given by,

$$
A_{j k}(\lambda, D)=\frac{E-D+1}{2 U} \frac{\partial U}{\partial \chi} \delta_{j k}+R_{j k}(\lambda, D) .
$$

\section{PROOF OF FUNDAMENTAL IDEAL MEMBERSHIP}

In this section we give a proof of the fundamental ideal membership (3.3). As explained in Sec. III, the latter implies that differential equations of the form in Eq. (1.2) can always be derived in Baikov representation without involving dimension-shift identities-i.e., relations between integrals in $D$ and $D-2$ dimensions of the form in Eq. (A4).

We emphasize that the proof is constructive and gives an explicit construction of the cofactors $\left(a_{1}, \ldots, a_{m}, b\right)$ in Eq. (3.2).

Theorem 1. Taking the Baikov polynomial in Eq. (2.13) to depend on the Baikov variables in Eq. (2.12) and the $\lambda_{i j}$ in Eq. (2.7) and letting $\chi$ denote any of the $\lambda_{i j}$, there exist polynomials $\left(a_{i}, b\right)$ such that the following relation holds,

$$
\frac{\partial F}{\partial \chi}=\sum_{i=1}^{m} a_{i} \frac{\partial F}{\partial z_{i}}+b F
$$

Proof.-Given a generic matrix $R=\left(r_{i j}\right)_{i, j=1, \ldots, n}$ whose entries are all independent, the Laplace expansion of the determinant along the $i$ th row can be expressed in the form,

$$
\left[\sum_{k=1}^{n} r_{j k} \frac{\partial(\operatorname{det} R)}{\partial r_{i k}}\right]-\delta_{i j} \operatorname{det} R=0, \quad 1 \leq i, j \leq n .
$$

The identities with $i \neq j$ arise by replacing the $i$ th row of $R$ by the $j$ th row, $r_{i k} \rightarrow r_{j k}$, which clearly produces a matrix with a vanishing determinant.

For a symmetric matrix $S=\left(s_{i j}\right)_{i, j=1, \ldots, n}$ the entries satisfy $s_{i j}=s_{j i}$ and thus are not independent, but rather linearly constrained. In this case, the Laplace expansion produces the identities,

$$
\left[\sum_{k=1}^{n}\left(1+\delta_{i k}\right) s_{j k} \frac{\partial(\operatorname{det} S)}{\partial s_{i k}}\right]-2 \delta_{i j} \operatorname{det} S=0, \quad 1 \leq i, j \leq n .
$$

In taking the derivatives we must bear in mind that the entries are not independent. We therefore imagine making the replacement $s_{j i} \rightarrow s_{i j}$ with $i \leq j$ in $S$ prior to taking derivatives, and in turn interpret $\frac{\partial(\operatorname{det} S)}{\partial s_{i k}}$ with $i>k$ as $\frac{\partial(\operatorname{det} S)}{\partial s_{k i}}$.

For the Gram matrix $S$ in Eq. (2.5), the identity (4.3) implies for $1 \leq i, j \leq E$ that, 


$$
\sum_{k=1}^{E}\left(1+\delta_{i k}\right) \lambda_{j k}\left(\frac{\partial F}{\partial \lambda_{i k}}\right)_{x}=2 \delta_{i j} F-\sum_{q=E+1}^{E+L}\left(1+\delta_{i q}\right) x_{j q} \frac{\partial F}{\partial x_{i q}} .
$$

The notation $\left(\frac{\partial F}{\partial \lambda_{i k}}\right)_{x}$ is used to emphasize that $F$ is here treated as a function of $x$ and $\lambda$, and the $x$-variables are treated as constants when evaluating the derivative. On the other hand, we may use the relations in Eqs. (2.11) and (2.12) to express $F$ as a function of $z$ and $\lambda$. The corresponding derivative obtained while treating the $z$-variables as constants is related to the former derivative through the chain rule,

$$
\left(\frac{\partial F}{\partial \lambda_{i k}}\right)_{x}=\left(\frac{\partial F}{\partial \lambda_{i k}}\right)_{z}+\sum_{\alpha=1}^{m} \frac{\partial z_{\alpha}}{\partial \lambda_{i k}} \frac{\partial F}{\partial z_{\alpha}} \quad \text { for } \quad 1 \leq i, k \leq E .
$$

Now, for a fixed $1 \leq i \leq E$, consider the left-hand side of Eq. (4.4) as the product of a matrix $\left(G_{i}\right)_{j k}$ and a vector $\left(\frac{\partial F}{\partial \lambda_{i k}}\right)_{x}$. That is, by defining for a fixed $1 \leq i \leq E$ the matrix,

$$
\left(G_{i}\right)_{j k}=\left(1+\delta_{i k}\right) \lambda_{j k} \text { for } 1 \leq j, k \leq E,
$$

we can recast Eq. (4.4) in the form,

$\sum_{k=1}^{E}\left(G_{i}\right)_{j k}\left(\frac{\partial F}{\partial \lambda_{i k}}\right)_{x}=2 \delta_{i j} F-\sum_{q=E+1}^{E+L}\left(1+\delta_{i q}\right) x_{j q} \frac{\partial F}{\partial x_{i q}}$.

From Eq. (4.6) we observe that $G_{i}$ is simply the Gram matrix $G$ in Eq. (2.8) with the $i$ th column multiplied by a factor of 2. This implies that $G_{i}$ is invertible, since $\operatorname{det} G_{i}=$ $2 \operatorname{det} G=2 U \neq 0$ for noncollinear external momenta.

Multiplying Eq. (4.7) by $G_{i}^{-1}$ from the left and using Eq. (4.5) and the chain rule,

$\frac{\partial F}{\partial x_{i q}}=\sum_{\alpha=1}^{m} \frac{\partial z_{\alpha}}{\partial x_{i q}} \frac{\partial F}{\partial z_{\alpha}} \quad$ for $\quad\left\{\begin{array}{l}1 \leq i \leq E, \\ E+1 \leq q \leq E+L,\end{array}\right.$

we find that,

$$
\left(\frac{\partial F}{\partial \lambda_{i k}}\right)_{z}=\left[\sum_{\alpha=1}^{m} a_{i k, \alpha} \frac{\partial F}{\partial z_{\alpha}}\right]+b_{i k} F
$$

where the cofactors are given by,

$$
\begin{aligned}
a_{i k, \alpha} & =-\frac{\partial z_{\alpha}}{\partial \lambda_{i k}}-\sum_{j=1}^{E} \sum_{q=E+1}^{E+L}\left(1+\delta_{i q}\right) \frac{\partial z_{\alpha}}{\partial x_{i q}}\left(G_{i}^{-1}\right)_{k j} x_{j q}, \\
b_{i k} & =2\left(G_{i}^{-1}\right)_{k i} .
\end{aligned}
$$

From the relations in Eqs. (2.11) and (2.12) it follows that the derivatives $\frac{\partial z_{\alpha}}{\partial \lambda_{i k}}$ and $\frac{\partial z_{\alpha}}{\partial x_{i q}}$ are integers. Furthermore, we may use the relations to express the $x$-variables as a linear combination of the $z$-variables. This proves the theorem and moreover shows that the cofactors $\left(a_{1}, \ldots, a_{m}, b\right)$ in Eq. (3.2) can be taken to be at most linear polynomials in the Baikov variables $z_{\alpha}$.

In the above proof, we assumed that all the $\lambda_{i j}$ are independent, as is the case for generic kinematics. For massless kinematics, the chain rules between independent Mandelstam invariants and $\lambda_{i j}$ imply that the conclusions about the fundamental ideal membership and the degrees of the cofactors continue to hold.

\section{DIFFERENTIAL EQUATIONS WITHOUT SQUARED PROPAGATORS}

In this section we investigate to what extent the IBP reduction formalisms of Refs. [46-48,59] are compatible with differential equations of the type in Eq. (1.2). As we will see, for a large class of multiloop diagrams, it is possible to avoid having the derivatives on the left-hand side of Eq. (1.2) produce integrals with squared propagators. We note that related work has appeared in Refs. $[59,61]$.

Returning to the derivation in Sec. III, from Eq. (3.6) we observe that terms with positive $\alpha_{i}$ will produce squared propagators for a generic polynomial $a_{i}$.

However, provided it is possible to choose the polynomials $a_{i}$ such that,

$$
a_{i}=z_{i} b_{i} \quad \text { for } \quad i=1, \ldots, k,
$$

where $b_{i}$ denote polynomials, the insertion (3.6) takes the following form,

$$
\begin{aligned}
Q_{j}= & -\sum_{i=1}^{k}\left[z_{i} \frac{\partial\left(b_{i} N_{j}\right)}{\partial z_{i}}+\left(1-\alpha_{i}\right) b_{i} N_{j}\right] \\
& -\sum_{i=k+1}^{m}\left[\frac{\partial\left(a_{i} N_{j}\right)}{\partial z_{i}}-\alpha_{i} \frac{a_{i} N_{j}}{z_{i}}\right]+\frac{D-L-E-1}{2} b N_{j} .
\end{aligned}
$$

We observe that the decomposition of $\frac{\partial F}{\partial \chi}$ in Eq. (3.2) with this choice of cofactors $a_{i}$ leads to a set of differential equations Eq. (3.5) without squared propagators.

In the following we will show that the enhanced ideal membership condition, equivalent to Eq. (3.2) combined with Eq. (5.1),

$$
\frac{\partial F}{\partial \chi} \in\left\langle z_{1} \frac{\partial F}{\partial z_{1}}, \ldots, z_{k} \frac{\partial F}{\partial z_{k}}, \frac{\partial F}{\partial z_{k+1}}, \ldots, \frac{\partial F}{\partial z_{m}}, F\right\rangle,
$$

turns out to hold for a large class of multiloop diagrams.

We note that ideal membership can be determined by computing a Gröbner basis $\mathcal{G}$ of the ideal on the right-hand side of Eq. (5.3) and computing the remainder $r$ of $\frac{\partial F}{\partial \chi}$ after polynomial division with respect to $\mathcal{G}$. Namely, Eq. (5.3) holds if and only if $r=0$. 
Alternatively, one can solve explicitly for the cofactors $\left(b_{1}, \ldots, b_{k}, a_{k+1}, \ldots, a_{m}, b\right)$ by starting with Ansätze which are linear in $\left(z_{1}, \ldots, z_{m}\right)$ and iteratively allowing for solutions of higher degree. This turns out to be an efficient approach in practice, as cofactors are found to be of low degrees and hence lead to linear systems of manageable sizes.

\section{EXAMPLES}

In this section we work out several examples of the formalism developed in Secs. III-V and present explicit results for the cofactors associated with the enhanced ideal membership (5.3) along with the resulting differential equations.

We display a selection of multiloop diagrams for which the enhanced ideal membership (5.3) has been verified to hold, and also show a counterexample.

\section{A. Massless planar double-box}

As a first simple example we consider the fully massless planar double-box diagram shown in Fig. 1.

In agreement with Eq. (2.12), we define the $z$-variables as follows, setting $P_{12} \equiv p_{1}+p_{2}$,

$$
\begin{aligned}
& z_{1}=\ell_{1}^{2}, \quad z_{2}=\left(\ell_{1}-p_{1}\right)^{2}, z_{3}=\left(\ell_{1}-P_{12}\right)^{2}, \\
& z_{4}=\left(\ell_{2}+P_{12}\right)^{2}, \quad z_{5}=\left(\ell_{2}-p_{4}\right)^{2}, \quad z_{6}=\ell_{2}^{2}, \\
& z_{7}=\left(\ell_{1}+\ell_{2}\right)^{2}, \quad z_{8}=\left(\ell_{1}+p_{4}\right)^{2}, \quad z_{9}=\left(\ell_{2}+p_{1}\right)^{2} \text {. }
\end{aligned}
$$

In terms of these variables and the Mandelstam invariants $s=\left(p_{1}+p_{2}\right)^{2}$ and $t=\left(p_{2}+p_{3}\right)^{2}$, the Gram determinant in Eq. (2.13) takes the following form,
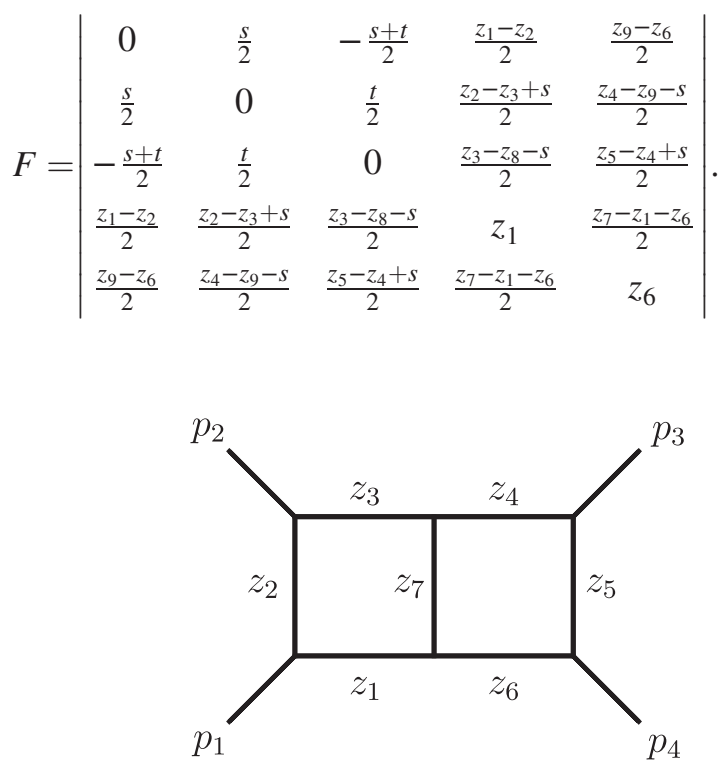

FIG. 1. The fully massless planar double-box diagram. All external momenta are taken to be outgoing.
We are interested in setting up differential equations for a basis of the vector space spanned by the diagram in Fig. 1 and its subdiagrams. An integral basis $\mathcal{I}$ can be obtained with AZURITE [74] which produces, setting $I_{\alpha} \equiv I(1 ; \alpha ; D)$ for brevity,

$$
\begin{aligned}
\mathcal{I}= & \left(s^{-1+2 \epsilon} I_{(0,1,0,0,1,0,1,0,0)}, s^{-1+2 \epsilon} I_{(1,0,0,1,0,0,1,0,0)},\right. \\
& s^{2 \epsilon} I_{(1,0,1,0,1,0,1,0,0)}, s^{2 \epsilon} I_{(1,0,1,1,0,1,0,0,0)}, \\
& s^{1+2 \epsilon} I_{(1,1,0,1,1,0,1,0,0)}, s^{1+2 \epsilon} I_{(1,1,1,0,1,0,1,0,0)}, \\
& \left.s^{3+2 \epsilon} I_{(1,1,1,1,1,1,1,0,0)}, s^{2+2 \epsilon} I_{(1,1,1,1,1,1,1,-1,0)}\right) .
\end{aligned}
$$

Here we rescaled the basis integrals $I_{\alpha}$ by appropriate factors of $s^{|\alpha|-4+2 \epsilon}$ to render them dimensionless. The basis integrals can thus only depend on the external kinematics through dimensionless ratios, of which only $\chi \equiv t / s$ is available.

We are therefore interested in differential equations for the basis integrals in Eq. (6.3) taken with respect to $\chi$. To this end, we set $t=\chi s$ in Eq. (6.2).

Now, in order to avoid introducing integrals with squared propagators in intermediate stages, we ask whether the enhanced ideal membership in Eq. (5.3) holds. We find that in the case at hand, a slightly stronger ideal membership holds,

$$
\frac{\partial F}{\partial \chi}=\sum_{i=1}^{9} b_{i} z_{i} \frac{\partial F}{\partial z_{i}}+b F .
$$

The cofactors are, setting $\mathbf{b}=\left(b_{1}, \ldots, b_{9}\right)$,

$$
\begin{aligned}
\mathbf{b}= & \left(\frac{z_{3}-z_{8}}{\chi(\chi+1) s}, \frac{z_{3}-z_{8}-\chi s-s}{\chi(\chi+1) s}, \frac{z_{3}-z_{8}-s}{\chi(\chi+1) s},\right. \\
& \frac{z_{4}-z_{5}-s}{\chi(\chi+1) s}, \frac{z_{4}-z_{5}-s}{\chi(\chi+1) s}, \frac{z_{4}-z_{5}}{\chi(\chi+1) s}, \\
& \left.\frac{z_{3}+z_{4}-z_{5}-z_{8}-s}{\chi(\chi+1) s}, \frac{z_{3}-z_{8}-s}{\chi(\chi+1) s}, \frac{z_{4}-z_{5}-\chi s-s}{\chi(\chi+1) s}\right) \\
b= & -\frac{2 z_{3}+2 z_{4}-2 z_{5}-2 z_{8}-2 \chi s-3 s}{\chi(\chi+1) s} .
\end{aligned}
$$

These cofactors were found by writing Ansätze for $\left(b_{i}, b\right)$ which are linear in $\left(z_{1}, \ldots, z_{9}\right)$ and solving the resulting linear system. Having obtained expressions for the cofactors, we insert them into Eq. (5.2) to obtain the explicit right-hand side of Eq. (3.5). After applying integration-byparts reductions to the resulting right-hand side, we find a system of differential equations of the desired form,

$$
\frac{\partial}{\partial \chi} \mathcal{I}(\chi, \epsilon)=A(\chi, \epsilon) \mathcal{I}(\chi, \epsilon),
$$

where we have set $\epsilon=\frac{4-D}{2}$, as we are interested in computing the integrals in a Laurent expansion around four space-time dimensions. The resulting coefficient 
matrix $A(\chi, \epsilon)$ is not particularly illuminating, and rather than presenting its explicit form, we take one further step [55] and rotate to a basis in which the coefficient matrix becomes proportional to $\epsilon$.

To this end, we note that under a change of integral basis,

$$
\mathcal{J}(\chi, \epsilon)=U(\chi, \epsilon) \mathcal{I}(\chi, \epsilon)
$$

the system of differential equations (6.6) becomes,

$$
\frac{\partial}{\partial \chi} \mathcal{J}(\chi, \epsilon)=\hat{A}(\chi, \epsilon) \mathcal{J}(\chi, \epsilon)
$$

where the transformed coefficient matrix is,

$$
\hat{A}=U A U^{-1}+\frac{\partial U}{\partial \chi} U^{-1}
$$

We can find a change-of-basis matrix $U$ with the desired property by using FUCHSIA [68]. Providing as input the coefficient matrix $A(\chi, \epsilon)$ computed in Eq. (6.6), it finds the following transformation matrix,

$$
\begin{aligned}
U= & \operatorname{diag}\left(\frac{(1-2 \epsilon)(1-3 \epsilon)(2-3 \epsilon)}{120 \epsilon^{3} \chi},\right. \\
& \frac{(1-2 \epsilon)(1-3 \epsilon)(-2+3 \epsilon)}{120 \epsilon^{3}}, \frac{(1-2 \epsilon)(1-3 \epsilon)}{24 \epsilon^{2}}, \\
& \left.\frac{(1-2 \epsilon)^{2}}{18 \epsilon^{2}},-\frac{\chi+1}{2}, \frac{-1+2 \epsilon}{6 \epsilon},-\frac{\chi}{2}, \frac{1}{2}\right)
\end{aligned}
$$

The transformed coefficient matrix is proportional to $\epsilon$, as desired, and takes the following form,

$$
\hat{A}=\epsilon\left(\frac{a_{0}}{\chi}+\frac{a_{-1}}{\chi+1}\right)
$$

where $a_{0}$ and $a_{-1}$ are matrices with integer entries,

$$
a_{0}=\left(\begin{array}{cccccccc}
-2 & 0 & 0 & 0 & 0 & 0 & 0 & 0 \\
0 & 0 & 0 & 0 & 0 & 0 & 0 & 0 \\
0 & 0 & 0 & 0 & 0 & 0 & 0 & 0 \\
0 & 0 & 0 & 0 & 0 & 0 & 0 & 0 \\
-60 & -60 & 0 & 0 & -2 & 0 & 0 & 0 \\
20 & 0 & -4 & 0 & 0 & -2 & 0 & 0 \\
-360 & 360 & 72 & 0 & 12 & 36 & -2 & 0 \\
540 & -360 & -90 & -9 & -18 & -36 & 1 & 1
\end{array}\right),
$$

and

$$
a_{-1}=\left(\begin{array}{cccccccc}
0 & 0 & 0 & 0 & 0 & 0 & 0 & 0 \\
0 & 0 & 0 & 0 & 0 & 0 & 0 & 0 \\
0 & 0 & 0 & 0 & 0 & 0 & 0 & 0 \\
0 & 0 & 0 & 0 & 0 & 0 & 0 & 0 \\
0 & 0 & 0 & 0 & 2 & 0 & 0 & 0 \\
-20 & 0 & 4 & 0 & 0 & 1 & 0 & 0 \\
360 & -720 & -36 & 18 & -12 & -36 & 2 & 2 \\
-540 & 360 & 90 & -9 & 18 & 36 & -1 & -1
\end{array}\right) .
$$

Thus, we have shown that it is possible to derive differential equations of the type (6.6) for the basis integrals in Eq. (6.3) and achieve a canonical form (6.11) of the system without introducing integrals with doubled propagators in intermediate stages.

\section{B. Massless nonplanar double-box diagram}

As a slightly more involved example, let us consider the fully massless nonplanar double-box diagram shown in Fig. 2.

We define the $z$-variables (2.12) as follows,

$$
\begin{aligned}
& z_{1}=\ell_{1}^{2}, \quad z_{2}=\left(\ell_{1}-p_{1}\right)^{2}, z_{3}=\left(\ell_{1}-P_{12}\right)^{2}, \\
& z_{4}=\left(\ell_{1}+\ell_{2}+p_{3}\right)^{2}, z_{5}=\left(\ell_{2}-p_{4}\right)^{2}, z_{6}=\ell_{2}^{2} \text {, } \\
& z_{7}=\left(\ell_{1}+\ell_{2}\right)^{2}, \quad z_{8}=\left(\ell_{1}+p_{4}\right)^{2}, \quad z_{9}=\left(\ell_{2}+p_{1}\right)^{2} \text {. }
\end{aligned}
$$

In terms of these variables and the Mandelstam invariants $s$ and $t$, the Gram determinant in Eq. (2.13) takes the following form,

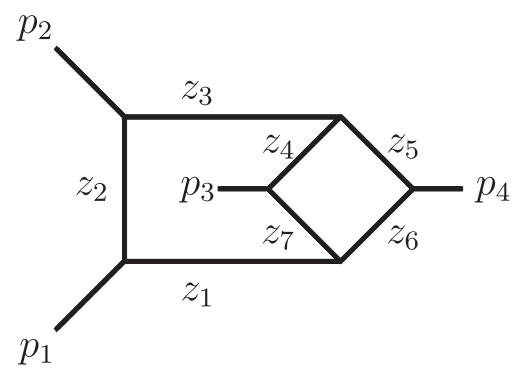

FIG. 2. The fully massless nonplanar double-box diagram. All external momenta are taken to be outgoing. 


$$
F=\left|\begin{array}{ccccc}
0 & \frac{s}{2} & -\frac{s+t}{2} & \frac{z_{1}-z_{2}}{2} & \frac{z_{9}-z_{6}}{2} \\
\frac{s}{2} & 0 & \frac{t}{2} & \frac{z_{2}-z_{3}+s}{2} & \frac{1}{2}\left(z_{3}-z_{4}+z_{5}+z_{7}-z_{8}-z_{9}-s\right) \\
-\frac{s+t}{2} & \frac{t}{2} & 0 & \frac{z_{3}-z_{8}-s}{2} & \frac{1}{2}\left(z_{4}-z_{3}-z_{7}+z_{8}+s\right) \\
\frac{z_{1}-z_{2}}{2} & \frac{z_{2}-z_{3}+s}{2} & \frac{z_{3}-z_{8}-s}{2} & z_{1} & \frac{z_{7}-z_{1}-z_{6}}{2} \\
\frac{z_{9}-z_{6}}{2} & \frac{1}{2}\left(z_{3}-z_{4}+z_{5}\right. & & & z_{6} \\
& \left.+z_{7}-z_{8}-z_{9}-s\right) & \frac{1}{2}\left(z_{4}-z_{3}-z_{7}+z_{8}+s\right) & \frac{z_{7}-z_{1}-z_{6}}{2} &
\end{array}\right|
$$

Our aim is again to write down differential equations for a basis of the vector space spanned by the diagram in Fig. 2 and its subdiagrams. AzURITE produces the following integral basis,

$$
\begin{aligned}
\mathcal{I}= & \left(s^{-1+2 \epsilon} I_{(1,0,0,1,1,0,0,0,0)}, \quad s^{-1+2 \epsilon} I_{(0,1,0,1,0,1,0,0,0)}, \quad s^{-1+2 \epsilon} I_{(0,1,0,0,1,0,1,0,0)}, \quad s^{2 \epsilon} I_{(1,0,1,1,0,1,0,0,0)},\right. \\
& s^{1+2 \epsilon} I_{(1,1,1,1,0,1,0,0,0)}, \quad s^{1+2 \epsilon} I_{(1,1,1,0,1,0,1,0,0)}, \quad s^{1+2 \epsilon} I_{(1,1,0,1,1,1,0,0,0)}, s^{1+2 \epsilon} I_{(1,1,0,1,1,0,1,0,0)}, \\
& \left.s^{1+2 \epsilon} I_{(0,1,0,1,1,1,1,0,0)}, \quad s^{2+2 \epsilon} I_{(1,0,1,1,1,1,1,0,0)}, \quad s^{2+2 \epsilon} I_{(1,1,1,1,1,1,1,-1,0)}, \quad s^{3+2 \epsilon} I_{(1,1,1,1,1,1,1,0,0)}\right),
\end{aligned}
$$

where we have again rescaled the basis integrals by appropriate powers of $s$ to ensure that they only depend on kinematics through $\chi$.

Our aim is now to write down differential equations for the basis integrals in Eq. (6.16) without encountering squared propagators in intermediate stages. We therefore ask whether the enhanced ideal membership in Eq. (5.3) holds. Once again, the "bonus" ideal membership (6.4) turns out to hold, with the cofactors,

$$
\begin{aligned}
\mathbf{b}= & \left(\frac{z_{3}-z_{8}}{\chi(\chi+1) s}, \frac{z_{3}-z_{8}-\chi s-s}{\chi(\chi+1) s}, \frac{z_{3}-z_{8}-s}{\chi(\chi+1) s}, \frac{2 z_{3}-z_{4}+z_{7}-2 z_{8}-s}{\chi(\chi+1) s}, \frac{z_{3}-z_{4}+z_{7}-z_{8}-s}{\chi(\chi+1) s}, \frac{z_{3}-z_{4}+z_{7}-z_{8}}{\chi(\chi+1) s},\right. \\
& \left.\frac{2 z_{3}-z_{4}+z_{7}-2 z_{8}-s}{\chi(\chi+1) s}, \frac{z_{3}-z_{8}-s}{\chi(\chi+1) s}, \frac{z_{3}-z_{4}+z_{7}-z_{8}-\chi s-s}{\chi(\chi+1) s}\right) \\
b= & -\frac{4 z_{3}-2 z_{4}+2 z_{7}-4 z_{8}-2 \chi s-3 s}{\chi(\chi+1) s} .
\end{aligned}
$$

We insert these expressions into Eq. (5.2) to find the right-hand side of Eq. (3.5) and then apply integration-by-parts reductions to the latter to find explicit differential equations of the form (6.6).

In order to present the resulting differential equations in a useful form, we use FUCHSIA to find a change-of-basis matrix $U(6.7)$ to a canonical representation. In the case at hand, we prefer to present the matrix inverse $U^{-1}$ which has a slightly more compact expression. The latter takes the following form,

$$
\left(U^{-1}\right)_{i j}=\delta_{i j} v_{1, i}+\delta_{i, 12} v_{2, j}
$$

where the vectors $v_{1}$ and $v_{2}$ are given by,

$$
\begin{aligned}
& v_{1}=\left(\frac{30 \epsilon^{3}(1+4 \epsilon)}{(1+\epsilon)(1-2 \epsilon)(1-3 \epsilon)(3 \epsilon-2)}, \frac{30 \epsilon^{3}(1+4 \epsilon)(\chi+1)}{(1+\epsilon)(1-2 \epsilon)(1-3 \epsilon)(2-3 \epsilon)}, \frac{30 \epsilon^{3}(1+4 \epsilon) \chi}{(1+\epsilon)(1-2 \epsilon)(1-3 \epsilon)(2-3 \epsilon)},\right. \\
& \frac{6 \epsilon^{2}(1+4 \epsilon)}{(1+\epsilon)(1-2 \epsilon)(1-3 \epsilon)}, \frac{3 \epsilon(1+4 \epsilon)}{2(1+\epsilon)(-1+2 \epsilon)}, \frac{3 \epsilon(1+4 \epsilon)}{2(1+\epsilon)(-1+2 \epsilon)},-\frac{1+4 \epsilon}{2 \chi(1+\epsilon)},-\frac{1+4 \epsilon}{2(\chi+1)(1+\epsilon)}, \\
&\left.\frac{1+4 \epsilon}{2(1+\epsilon)}, \frac{1+4 \epsilon}{2(1+\epsilon)},-\frac{1+4 \epsilon}{2(\chi+1)(1+\epsilon)},-\frac{1+4 \epsilon}{2 \chi(1+\epsilon)}\right), \\
& v_{2}=\left(\frac{180 \epsilon}{\chi(\chi+1)(1+\epsilon)}, \frac{60}{\chi}, \frac{60(\chi+1+\chi \epsilon+4 \epsilon)}{\chi(\chi+1)(1+\epsilon)}, 0,0,0,-\frac{2(\chi+1+\chi \epsilon+4 \epsilon)}{\chi(\chi+1)(1+\epsilon)},-\frac{2}{\chi},-\frac{6.19)}{\chi(\chi+1)(1+\epsilon)}, 0, \frac{6 \epsilon}{2(\chi+1)(1+\epsilon)}, 0\right) .
\end{aligned}
$$


The transformed coefficient matrix takes the following form,

$$
\hat{A}=\epsilon\left(\frac{a_{0}}{\chi}+\frac{a_{-1}}{\chi+1}\right),
$$

where $a_{0}$ and $a_{-1}$ are matrices with integer entries,

$$
a_{0}=\left(\begin{array}{cccccccccccc}
0 & 0 & 0 & 0 & 0 & 0 & 0 & 0 & 0 & 0 & 0 & 0 \\
0 & 0 & 0 & 0 & 0 & 0 & 0 & 0 & 0 & 0 & 0 & 0 \\
0 & 0 & -2 & 0 & 0 & 0 & 0 & 0 & 0 & 0 & 0 & 0 \\
0 & 0 & 0 & 0 & 0 & 0 & 0 & 0 & 0 & 0 & 0 & 0 \\
0 & 20 & 0 & 4 & 1 & 0 & 0 & 0 & 0 & 0 & 0 & 0 \\
0 & 0 & 20 & -4 & 0 & -2 & 0 & 0 & 0 & 0 & 0 & 0 \\
0 & 0 & 0 & 0 & 0 & 0 & 2 & 0 & 0 & 0 & 0 & 0 \\
-60 & 0 & -60 & 0 & 0 & 0 & 0 & -2 & 0 & 0 & 0 & 0 \\
0 & -60 & -60 & 0 & 0 & 0 & 0 & 0 & -2 & 0 & 0 & 0 \\
0 & 0 & 0 & 0 & 0 & 0 & 0 & 0 & 0 & 0 & 0 & 0 \\
-360 & -60 & 660 & -36 & 0 & -18 & -4 & -16 & -6 & 1 & 1 & -1 \\
-120 & 420 & 420 & -36 & 0 & -18 & -16 & -12 & 6 & 0 & 0 & -2
\end{array}\right),
$$

and

$$
a_{-1}=\left(\begin{array}{cccccccccccc}
0 & 0 & 0 & 0 & 0 & 0 & 0 & 0 & 0 & 0 & 0 & 0 \\
0 & -2 & 0 & 0 & 0 & 0 & 0 & 0 & 0 & 0 & 0 & 0 \\
0 & 0 & 0 & 0 & 0 & 0 & 0 & 0 & 0 & 0 & 0 & 0 \\
0 & 0 & 0 & 0 & 0 & 0 & 0 & 0 & 0 & 0 & 0 & 0 \\
0 & -20 & 0 & -4 & -2 & 0 & 0 & 0 & 0 & 0 & 0 & 0 \\
0 & 0 & -20 & 4 & 0 & 1 & 0 & 0 & 0 & 0 & 0 & 0 \\
60 & -60 & 0 & 0 & 0 & 0 & -2 & 0 & 0 & 0 & 0 & 0 \\
0 & 0 & 0 & 0 & 0 & 0 & 0 & 2 & 0 & 0 & 0 & 0 \\
0 & 60 & 60 & 0 & 0 & 0 & 0 & 0 & -2 & 0 & 0 & 0 \\
0 & 0 & 0 & 0 & 0 & 0 & 0 & 0 & 0 & 0 & 0 & 0 \\
-360 & -180 & -180 & -36 & -18 & 0 & 12 & 0 & 6 & 0 & -2 & 0 \\
-600 & -660 & 60 & -36 & -18 & 0 & 24 & -4 & -6 & 1 & -1 & 1
\end{array}\right) .
$$

We conclude that it is possible to derive differential equations for the basis integrals in Eq. (6.16) of the type (6.6) and achieve a canonical form (6.21) of the system without introducing integrals with doubled propagators in intermediate stages.

\section{Further verification of the enhanced ideal membership}

We have verified that the enhanced ideal membership in Eq. (5.3) holds in many multiloop examples. Rather than writing down the explicit form of the resulting differential 

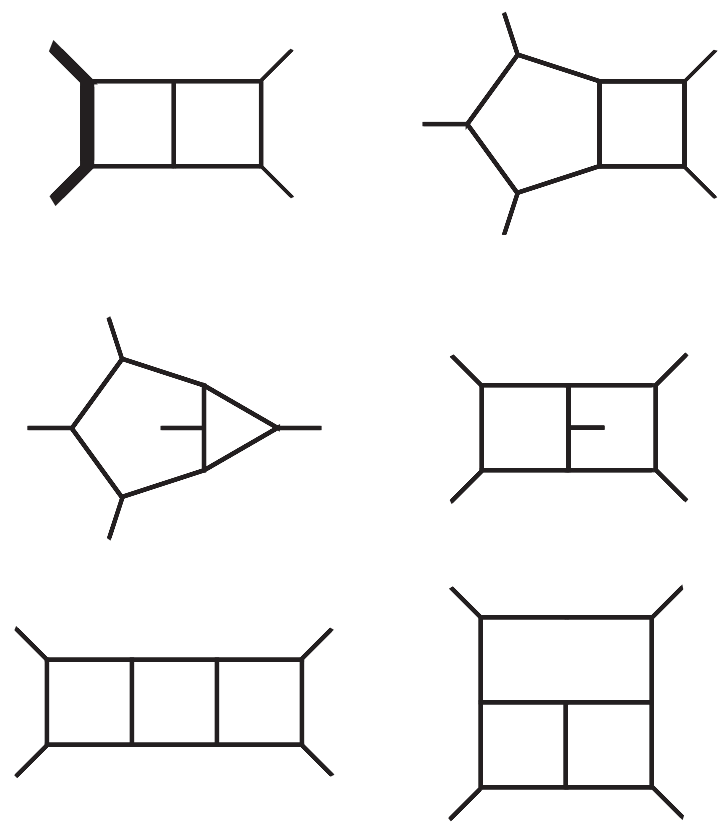

FIG. 3. A selection of diagrams for which the enhanced ideal membership in Eq. (5.3) has been verified. The bold lines represent massive momenta and propagators.

equations, in this section we restrict ourselves to displaying in Fig. 3 a selection of diagrams for which Eq. (5.3) was verified to hold.

As explained above, we note that Eq. (5.3) implies that we can derive differential equations of the type (1.2) without introducing integrals with doubled propagators in intermediate stages.

\section{Counterexample of the enhanced ideal membership}

Although the enhanced ideal membership in Eq. (5.3) holds true for a large class of Feynman diagrams, we have found that it is not a general property of the Baikov polynomial $F$.

In particular, consider the diagram displayed in Fig. 4. Upon computing a Gröbner basis $\mathcal{G}$ of the ideal and performing polynomial division of $\frac{\partial F}{\partial \chi}$ with respect to $\mathcal{G}$, one finds a nonvanishing remainder. Hence, the diagram in Fig. 4 provides a counterexample to the enhanced ideal membership in Eq. (5.3).

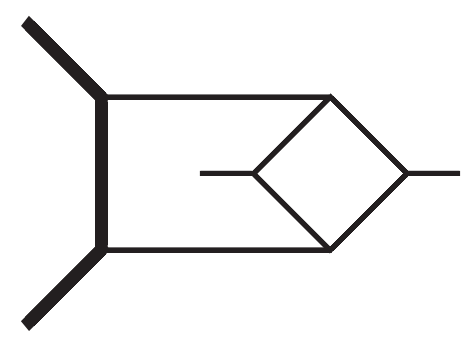

FIG. 4. Nonplanar double-box diagram. The bold lines represent massive momenta and propagators. For this diagram, the enhanced ideal membership Eq. (5.3) does not hold.

\section{CONCLUSIONS}

In this paper we have studied differential equations for loop integrals in Baikov representation. We gave a proof that the Baikov polynomial satisfies the ideal membership property (3.3). We have shown that, as a result, differential equations can be derived without involving dimension-shift identities. This is advantageous because the latter are computationally intensive to generate, as they require integration-by-parts identities that involve integrals with high powers of loop momentum monomials. We remark that the proof is constructive and gives an explicit construction of the cofactors.

We have moreover shown that an enhanced ideal membership property (5.3) holds for a large class of multiloop diagrams. As a result, differential equations of the type (1.2) can be derived without introducing integrals with doubled propagators in intermediate stages. This is beneficial because it limits the set of integrals for which integration-by-parts reductions must be determined.

At the same time, we have identified a counterexample to Eq. (5.3), showing that it is not a general property of the Baikov polynomial. An interesting open problem is therefore to classify the diagrams for which the enhanced ideal membership property (5.3) holds.

We emphasize that Eq. (5.3) holds for highly nontrivial loop diagrams whose differential equations are not attainable with standard methods. It therefore appears to be a promising tool for the calculation of multiloop integrals.

\section{ACKNOWLEDGMENTS}

We thank Roman N. Lee for Collaboration at an early stage of this work, in particular for his idea of proving Eq. (3.3) via Laplace expansion of the determinant of symmetric matrices. We also thank Hjalte Frellesvig, Harald Ita, David A. Kosower, Erik Panzer, Costas Papadopoulos, Vladimir A. Smirnov and Mao Zeng for useful discussions. The research leading to these results has received funding from Swiss National Science Foundation (Ambizione Grant No. PZ00P2 161341) and funding from the European Research Council (ERC) under the European Union's Horizon 2020 research and innovation programme (Grant agreement No. 725110). The work of Y.Z. is also partially supported by the Swiss National Science Foundation through the NCCR SwissMap, Grant No. 141869. The work of J. B. is supported by Grant no. 615203 from the European Research Council under the FP7 and by the Swiss National Science Foundation through the NCCR SwissMAP. The work of K. J. L. is supported by ERC-2014-CoG, Grant No. 648630 IQFT.

\section{APPENDIX: DIFFERENTIAL EQUATIONS FROM DIMENSION-SHIFT IDENTITIES}

In this Appendix we work out the form of the differential equations (3.1) when $\frac{\partial F}{\partial \chi}$ is left unchanged. As discussed 
below this equation, the $\frac{1}{F}$ factor in the second line has the effect of shifting the space-time dimension from $D$ to $D-2$. Accordingly, we will show how to derive dimension-shift identities relating the latter two. We remark that in this approach, integrals with squared propagators are not generated in intermediate stages. It is therefore entirely compatible with the IBP reduction formalisms of Refs. [46-48,59].

It will be more convenient for the presentation to consider a $(D+2)$-dimensional integral. Taking the Baikov representation (2.14) of the latter and applying a derivative with respect to any external invariant $\chi$ we find,

$$
\begin{aligned}
& \frac{\partial}{\partial \chi} I_{j}\left(N_{j} ; \alpha ; D+2\right) \\
& =\frac{2^{L-1} \Gamma(D-L-E+2)}{\Gamma(D-E+1) U} I_{j}\left(N_{j} \frac{\partial F}{\partial \chi} ; \alpha ; D\right) \\
& \quad+\frac{E-D-1}{2 U} \frac{\partial U}{\partial \chi} I_{j}\left(N_{j} ; \alpha ; D+2\right) .
\end{aligned}
$$

We remark that, in computing the ratio $\frac{C_{E}^{L}(D+2)}{C_{E}^{L}(D)}$ above, we used the fact that the entries of $A$ [defined in Eq. (2.11)] are integers which are independent of $D$. Hence $\operatorname{det} A$ cancels out of the ratio, cf. Eq. (2.15).

Our aim is now to reexpress the $(D+2)$-dimensional integrals in Eq. (A1) in terms of $D$-dimensional integrals. To determine the requisite dimension-shift identities [52,75-78], we start by observing that,

$$
\begin{aligned}
I_{j}\left(N_{j} ; \alpha ; D+2\right) & =\frac{C_{E}^{L}(D+2)}{C_{E}^{L}(D) U} I_{j}\left(F N_{j} ; \alpha ; D\right) \\
& =\frac{2^{L} \Gamma(D-L-E+1)}{\Gamma(D-E+1) U} I_{j}\left(F N_{j} ; \alpha ; D\right) .
\end{aligned}
$$

Now, by applying integration-by-parts reductions to the right-hand side of Eq. (A3), we can reexpress $I_{j}\left(F N_{j} ; \alpha ; D\right)$ as a linear combination of the basis integrals. In this way we find,

$$
I_{j}\left(N_{j} ; \alpha ; D+2\right)=\sum_{k=1}^{M} T_{j k}(\chi) I_{k}\left(N_{k} ; \alpha ; D\right) .
$$

Applying $\frac{\partial}{\partial \chi}$ to Eq. (A4) and comparing the resulting expression for $\frac{\partial}{\partial \chi} I_{j}\left(N_{j} ; \alpha ; D+2\right)$ with Eq. (A1) we find, after applying Eq. (A4) and multiplying by $\left(T^{-1}\right)_{i j}$ from the left,

$$
\frac{\partial}{\partial \chi} I_{i}\left(N_{i} ; \alpha ; D\right)=\sum_{k=1}^{M} A_{i k} I_{k}\left(N_{k} ; \alpha ; D\right),
$$

where the coefficient matrix is given by,

$$
\begin{aligned}
A_{i k}= & \sum_{j=1}^{M}\left(T^{-1}\right)_{i j}\left[\frac{2^{L-1} \Gamma(D-L-E+2)}{\Gamma(D-E+1) U} R_{j k}-\frac{\partial T_{j k}(\chi)}{\partial \chi}\right] \\
& +\frac{E-D-1}{2 U} \frac{\partial U}{\partial \chi} \delta_{i k},
\end{aligned}
$$

and where the matrix $R$ arises as the matrix of IBP reduction coefficients,

$$
I_{j}\left(N_{j} \frac{\partial F}{\partial \chi} ; \alpha ; D\right)=\sum_{k=1}^{M} R_{j k} I_{k}\left(N_{k} ; \alpha ; D\right) .
$$

The above dimension-shift approach has the virtue that it avoids generating integrals with squared propagators in intermediate stages. It is therefore amenable to the IBP reduction formalisms of Refs. [46-48,59]. However, performing the integration-by-parts reductions of the righthand side of Eq. (A7) is computationally intensive in practice. This is because the Baikov polynomial $F$ has higher powers of monomials than encountered in the Feynman rules of gauge theory, and as a result Gaussian elimination must be applied to large linear systems.
[1] R. Bonciani, A. Ferroglia, T. Gehrmann, D. Maitre, and C. Studerus, J. High Energy Phys. 07 (2008) 129.

[2] T. Gehrmann and E. W. N. Glover, Phys. Lett. B 676, 146 (2009).

[3] R. Bonciani, A. Ferroglia, T. Gehrmann, and C. Studerus, J. High Energy Phys. 08 (2009) 067.

[4] R. Bonciani, A. Ferroglia, T. Gehrmann, A. von Manteuffel, and C. Studerus, J. High Energy Phys. 01 (2011) 102.

[5] T. Gehrmann and L. Tancredi, J. High Energy Phys. 02 (2012) 004.
[6] T. Gehrmann, M. Jaquier, E. W. N. Glover, and A. Koukoutsakis, J. High Energy Phys. 02 (2012) 056.

[7] T. Gehrmann, L. Tancredi, and E. Weihs, J. High Energy Phys. 04 (2013) 101.

[8] A. von Manteuffel and C. Studerus, J. High Energy Phys. 10 (2013) 037.

[9] T. Gehrmann, L. Tancredi, and E. Weihs, J. High Energy Phys. 08 (2013) 070.

[10] J. M. Henn and V. A. Smirnov, J. High Energy Phys. 11 (2013) 041. 
[11] R. Bonciani, A. Ferroglia, T. Gehrmann, A. von Manteuffel, and C. Studerus, J. High Energy Phys. 12 (2013) 038.

[12] J. M. Henn, K. Melnikov, and V. A. Smirnov, J. High Energy Phys. 05 (2014) 090.

[13] T. Gehrmann, A. von Manteuffel, L. Tancredi, and E. Weihs, J. High Energy Phys. 06 (2014) 032.

[14] F. Caola, J. M. Henn, K. Melnikov, and V. A. Smirnov, J. High Energy Phys. 09 (2014) 043.

[15] F. Caola, J. M. Henn, K. Melnikov, A. V. Smirnov, and V. A. Smirnov, J. High Energy Phys. 11 (2014) 041.

[16] C. G. Papadopoulos, D. Tommasini, and C. Wever, J. High Energy Phys. 01 (2015) 072.

[17] T. Huber and S. Kränkl, J. High Energy Phys. 04 (2015) 140.

[18] T. Gehrmann, A. von Manteuffel, and L. Tancredi, J. High Energy Phys. 09 (2015) 128.

[19] F. Caola, J. M. Henn, K. Melnikov, A. V. Smirnov, and V. A. Smirnov, J. High Energy Phys. 06 (2015) 129.

[20] A. von Manteuffel and L. Tancredi, J. High Energy Phys. 06 (2015) 197.

[21] R. Bonciani, S. Di Vita, P. Mastrolia, and U. Schubert, J. High Energy Phys. 09 (2016) 091.

[22] R. Bonciani, V. Del Duca, H. Frellesvig, J. M. Henn, F. Moriello, and V.A. Smirnov, J. High Energy Phys. 12 (2016) 096.

[23] K. Melnikov, L. Tancredi, and C. Wever, Phys. Rev. D 95, 054012 (2017).

[24] P. Mastrolia, M. Passera, A. Primo, and U. Schubert, J. High Energy Phys. 11 (2017) 198.

[25] M. Becchetti and R. Bonciani, J. High Energy Phys. 01 (2018) 048.

[26] S. Badger, H. Frellesvig, and Y. Zhang, J. High Energy Phys. 12 (2013) 045.

[27] S. Badger, G. Mogull, A. Ochirov, and D. O'Connell, J. High Energy Phys. 10 (2015) 064.

[28] T. Gehrmann, J. M. Henn, and N. A. Lo Presti, Phys. Rev. Lett. 116, 062001 (2016); 116, 189903(E) (2016).

[29] D. C. Dunbar and W. B. Perkins, Phys. Rev. D 93, 085029 (2016).

[30] D. C. Dunbar, G. R. Jehu, and W. B. Perkins, Phys. Rev. D 93, 125006 (2016).

[31] D. C. Dunbar and W. B. Perkins, Phys. Rev. Lett. 117, 061602 (2016).

[32] S. Badger, G. Mogull, and T. Peraro, J. High Energy Phys. 08 (2016) 063.

[33] D. C. Dunbar, J. H. Godwin, G. R. Jehu, and W. B. Perkins, Phys. Rev. D 96, 116013 (2017).

[34] S. Badger, C. Brønnum-Hansen, H. B. Hartanto, and T. Peraro, Phys. Rev. Lett. 120, 092001 (2018).

[35] C. G. Papadopoulos, D. Tommasini, and C. Wever, J. High Energy Phys. 04 (2016) 078.

[36] A. V. Smirnov and A. V. Petukhov, Lett. Math. Phys. 97, 37 (2011).

[37] S. Laporta, Phys. Lett. B 504, 188 (2001).

[38] S. Laporta, Int. J. Mod. Phys. A 15, 5087 (2000).

[39] C. Anastasiou and A. Lazopoulos, J. High Energy Phys. 07 (2004) 046.

[40] A. V. Smirnov, J. High Energy Phys. 10 (2008) 107.

[41] A. V. Smirnov, Comput. Phys. Commun. 189, 182 (2015).
[42] C. Studerus, Comput. Phys. Commun. 181, 1293 (2010).

[43] A. von Manteuffel and C. Studerus, arXiv:1201.4330.

[44] R. N. Lee, arXiv:1212.2685.

[45] P. Maierhoefer, J. Usovitsch, and P. Uwer, arXiv: 1705.05610.

[46] J. Gluza, K. Kajda, and D. A. Kosower, Phys. Rev. D 83, 045012 (2011).

[47] H. Ita, Phys. Rev. D 94, 116015 (2016).

[48] K. J. Larsen and Y. Zhang, Phys. Rev. D 93, 041701 (2016).

[49] A. von Manteuffel and R. M. Schabinger, Phys. Lett. B 744, 101 (2015).

[50] A. V. Kotikov, Phys. Lett. B 254, 158 (1991).

[51] A. V. Kotikov, Phys. Lett. B 267, 123 (1991).

[52] Z. Bern, L. J. Dixon, and D. A. Kosower, Nucl. Phys. B412, 751 (1994).

[53] E. Remiddi, Nuovo Cimento Soc. Ital. Fis. A110, 1435 (1997).

[54] T. Gehrmann and E. Remiddi, Nucl. Phys. B580, 485 (2000).

[55] J. M. Henn, Phys. Rev. Lett. 110, 251601 (2013).

[56] C. G. Papadopoulos, J. High Energy Phys. 07 (2014) 088.

[57] J. Ablinger, A. Behring, J. Blümlein, A. De Freitas, A. von Manteuffel, and C. Schneider, Comput. Phys. Commun. 202, 33 (2016).

[58] X. Liu, Y.-Q. Ma, and C.-Y. Wang, Phys. Lett. B 779, 353 (2018).

[59] Z. Bern, M. Enciso, H. Ita, and M. Zeng, Phys. Rev. D 96, 096017 (2017).

[60] H. Frellesvig and C. G. Papadopoulos, J. High Energy Phys. 04 (2017) 083.

[61] M. Zeng, J. High Energy Phys. 06 (2017) 121.

[62] M. Harley, F. Moriello, and R. M. Schabinger, J. High Energy Phys. 06 (2017) 049.

[63] J. Moser, Math. Z. 72, 379 (1959).

[64] R. N. Lee, J. High Energy Phys. 04 (2015) 108.

[65] R. N. Lee and A. A. Pomeransky, arXiv:1707.07856.

[66] C. Meyer, J. High Energy Phys. 04 (2017) 006.

[67] M. Prausa, Comput. Phys. Commun. 219, 361 (2017).

[68] O. Gituliar and V. Magerya, Comput. Phys. Commun. 219, 329 (2017).

[69] C. Meyer, Comput. Phys. Commun. 222, 295 (2018).

[70] P. A. Baikov, Phys. Lett. B 385, 404 (1996).

[71] The consistency of the Baikov representation in Eq. (2.14) with that used in Ref. [48] follows from the identity $\operatorname{det}_{i, j=1, \ldots, L} \mu_{i j}=\frac{F}{U}$, which in turn is a consequence of the Schur complement theorem in linear algebra. Also note that Ref. [48] uses the four-dimensional helicity scheme and therefore requires that the external momenta span a vector space of dimension at most four, i.e. $\operatorname{dim} \operatorname{span}\left\{p_{1}, \ldots, p_{n}\right\} \leq 4$. As a result, the exponent of the Baikov polynomial there, with $L=2$, takes the values $\frac{D-7}{2}$ and $\frac{D-6}{2}$ for $E \geq 4$ and $E=3$, respectively.

[72] We remark that the domain of integration in Eq. (2.14) depends on $\chi$. Hence one expects a term corresponding to the domain dependence on $\chi$ to appear in the derivative. However, this term vanishes since the integrand is zero on the boundary of the domain. We refer to Sec. 2 of Ref. [73] for a discussion of this point.

[73] J. Bosma, M. Sogaard, and Y. Zhang, J. High Energy Phys. 08 (2017) 051. 
[74] A. Georgoudis, K. J. Larsen, and Y. Zhang, Comput. Phys. Commun. 221, 203 (2017).

[75] Z. Bern, L. J. Dixon, and D. A. Kosower, Phys. Lett. B 302, 299 (1993); 318, 649(E) (1993).
[76] O. V. Tarasov, Phys. Rev. D 54, 6479 (1996).

[77] R. N. Lee, Nucl. Phys. B830, 474 (2010).

[78] R. N. Lee, Nucl. Phys. B, Proc. Suppl. 205-206, 135 (2010). 